\title{
Captação da Gestante para Pré-natal precoce
}

\section{Capture of the pregnant woman for early prenatal}

\section{Captación de la gestante para prenatal precoz}

\author{
MAndréa Ferreira Serrazina ${ }^{1}$, Geísa Sereno Velloso da Silva ${ }^{2}$
}

Como citar esse artigo. Serrazina, MF; da Silva, GSV. Captação da Gestante para Pré-natal precoce. Revista PróUniverSUS. 2019 Jan./Jun.; 10 (1): 2934

\section{Resumo}

O presente trabalho traz um panorama da assistência pré-natal no contexto da atenção básica de saúde e da Estratégia de Saúde da Família (ESF), alertando para a importância do profissional de enfermagem nesse processo para a eficácia da estratégia de saúde, da prevenção de doenças e da diminuição dos índices de mortalidade durante a gravidez. Tem como objetivos identificar as dificuldades do enfermeiro da atenção básica para a captação de gestantes no pré-natal do primeiro trimestre gestacional. Elaborou-se uma pesquisa de campo, de caráter descritivo e abordagem quali-quantitativa sobre as dificuldades vivenciadas pelo enfermeiro na atenção básica de saúde durante a assistência pré-natal, por meio de uma entrevista semi-estruturada realizada com 10 enfermeiros de diferentes unidades de saúde do município de Vassouras/RJ. Verificou-se a necessidade não só de ampliar a captação de gestantes para o recebimento desse cuidado como também a necessidade de aprimorar as estratégias utilizadas nas gestantes captadas. Concluiu-se que os enfermeiros da atenção básica em Vassouras passam por muitas dificuldades na captação de gestantes no primeiro trimestre gestacional, dificuldades inclusive de percepção da própria realidade e de formação e que o quadro não é pior por conta do papel exercido pelas agentes comunitárias de saúde nesse processo.

Palavras-chave: Assistência pré-natal, Atenção Básica, Enfermagem.

\begin{abstract}
The present study presents an overview of prenatal care in the context of basic health care and the Family Health Strategy (ESF), alerting the importance of the nursing professional in this process for the effectiveness of the health strategy, the prevention of disease and reduction of mortality rates during pregnancy. It aims to identify the difficulties of the primary care nurse for the capture of pregnant women in the prenatal period of the first gestational trimester. A descriptive and field-based qualitative and qualitative research was developed on the difficulties experienced by nurses in basic health care during prenatal care, through a semi-structured interview conducted with 10 nurses from different countries health units of the municipality of Vassouras / RJ. There was a need not only to increase the uptake of pregnant women to receive this care but also the need to improve the strategies used in pregnant women. It was concluded that primary care nurses in Vassouras face many difficulties in capturing pregnant women in the first trimester of pregnancy, difficulties including perception of their own reality and training, and that the picture is not worse because of the role played by the agents in this process.
\end{abstract}

Keywords: Prenatal care, Basic Attention, Nursing.

\section{Resumen}

The present study presents an overview of prenatal care in the context of basic health care and the Family Health Strategy (ESF), alerting the importance of the nursing professional in this process for the effectiveness of the health strategy, the prevention of disease and reduction of mortality rates during pregnancy. It aims to identify the difficulties of the primary care nurse for the capture of pregnant women in the prenatal period of the first gestational trimester. A descriptive and field-based qualitative and qualitative research was developed on the difficulties experienced by nurses in basic health care during prenatal care, through a semi-structured interview conducted with 10 nurses from different countries health units of the municipality of Vassouras / RJ. There was a need not only to increase the uptake of pregnant women to receive this care but also the need to improve the strategies used in pregnant women. It was concluded that primary care nurses in Vassouras face many difficulties in capturing pregnant women in the first trimester of pregnancy, difficulties including perception of their own reality and training, and that the picture is not worse because of the role played by the agents in this process.

Palabras clave: Prenatal Care, Básico de atención, Enfermería.

Afiliação dos autores: 1. Acadêmico do Curso de Enfermagem, Universidade de Vassouras. RJ, Brasil.

2. Mestre. Professor do Curso de Enfermagem, Universidade de Vassouras. RJ, Brasil. 


\section{Introdução}

A assistência ao pré-natal acontece a partir de várias ações dos serviços de saúde ${ }^{1}$, visando promover e prevenir de forma ampla a saúde do binômio mãe e filho, utilizando um protocolo padrão, sendo isto possível, à partir de mudanças nas políticas públicas onde se entendeu o cuidado como ação preventiva que diminua riscos ou agravos a essa gestação, evitando mortes materno-infantil.

O pré-natal é uma das assistências oferecidas também pelos enfermeiros na Atenção Básica (AB), e tem por finalidade garantir o melhor estado de saúde possível para a mãe e para o bebê, destacandose como fator precípuo na proteção e na prevenção a eventos danosos à saúde da gestante.

$\mathrm{O}$ presente estudo almeja contemplar identificar dificuldades que os enfermeiros enfrentam para a captação da gestante no primeiro trimestre gestacional, conhecer as estratégias usadas pelos Enfermeiros para buscar a gestante e implementar ações que tragam a mesma à consulta de pré-natal precoce.

\section{Metodologia}

A metodologia aplicada neste estudo tem caráter descritivo e abordagem quali-quantitativa, na tentativa de enxergar melhor o quadro atual da prática da assistência pré-natal na Estratégia de Saúde da Família do município de Vassouras e investigar as dificuldades enfrentadas pelos enfermeiros na captação das gestantes bem como as estratégias utilizadas por eles para lidar com essa questão.

A pesquisa foi realizada nas Unidades de Saúde da Cidade de Vassouras, no interior do Estado do Rio de Janeiro, onde os sujeitos da pesquisa serão 10 Enfermeiras das Estratégias de Saúde da Família e que atuam diretamente à consulta pré-natal, onde estarão respondendo a um questionário semi-estruturado, seguindo um roteiro pré-elaborado com questões que possam colher informações referentes à formação profissional, qualificação e experiências das enfermeiras que assistem a gestante na Unidade de Saúde. Tendo uma duração de 3(três) meses.

A Coleta de Dados foi realizada no mês de setembro de 2018 após aprovação do Comitê de Ética em Pesquisa da Universidade de Vassouras (CAAE: 92394218.8.0000.5290), através de uma entrevista semi-estruturada contendo 18 perguntas abertas e fechadas relacionadas ao tema (anexo 2). A entrevista foi realizada em Unidades de Saúde do município de Vassouras. Os dados coletados tratam indiretamente de um universo de 10 profissionais da enfermagem.

\section{Resultados e discussão}

Metade dos enfermeiros entrevistados não possuía nenhuma especialização até a data de realização da entrevista. A outra metade possuía especializações como Saúde da Família, UTI e Saúde coletiva. Uma pequena parcela, de $40 \%$ apenas, possuía especialização nos temas que tem relação direta com esse estudo. 10\% em Enfermagem Neonatal, 10\% em Obstetrícia, 10\% em Ginecologia.

Gráfico 1. Sexo dos profissionais de enfermagem

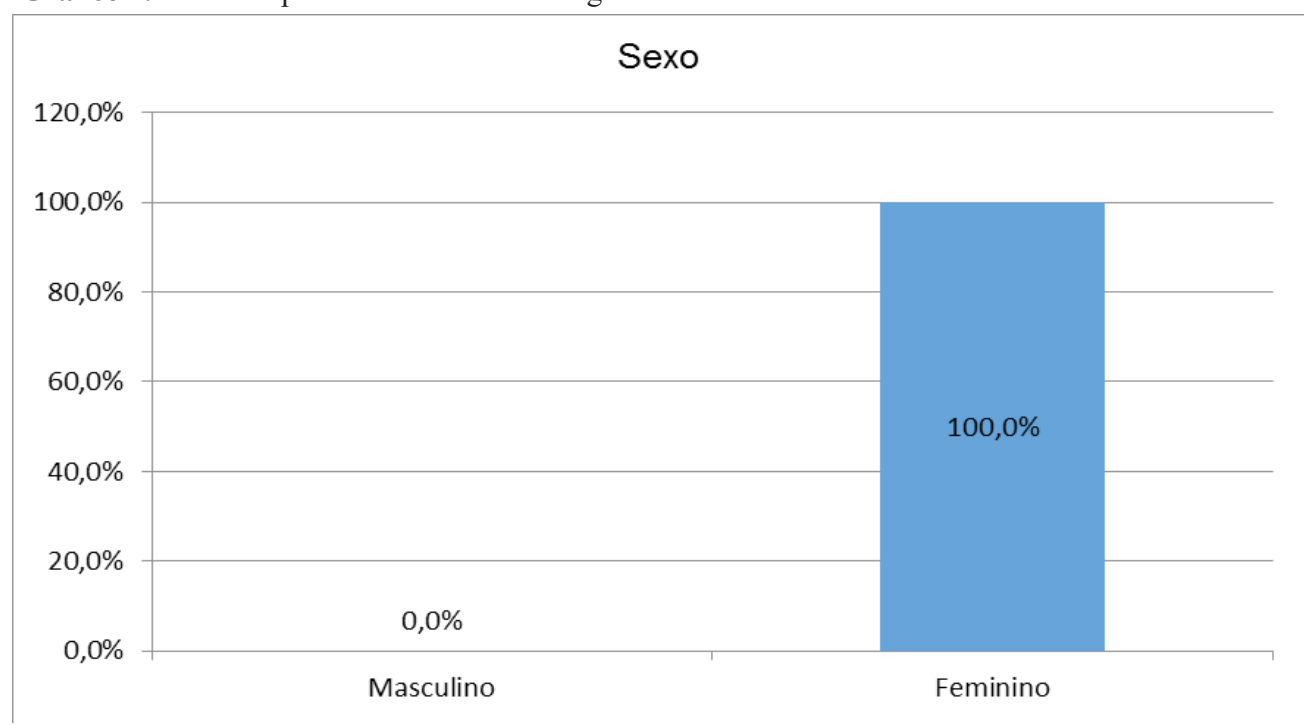

Fonte: Pesquisa do autor, 2018. 
O universo de entrevistados desta pesquisa foi composto em sua totalidade por profissionais da enfermagem atuantes na Atenção Básica de Saúde. Do total de entrevistados, $100 \%$ pertence ao sexo feminino dos quais a metade (50\%) tem entre 30 e 39 anos, $33 \%$ entre 20 e 29 anos, $8,5 \%$ entre 40 e 49 anos e $8,5 \%$ com idade entre 50 e 59 anos.

A primeira informação relevante do ponto de vista qualitativo e que norteia algumas hipóteses desse trabalho é a adesão ao pré-natal durante o primeiro trimestre gestacional. Muito abaixo do esperado, o índice médio de adesão à assistência pré-natal atual, levando-se em conta o total de todas as unidades é de $72 \%$. Variando entre míseros $33 \%$ na unidade com menor adesão até a cobertura completa, $100 \%$ na unidade de maior adesão. Ressaltando-se que o mínimo aceitável é 80\%.

Gráfico 2. Idade dos profissionais de enfermagem

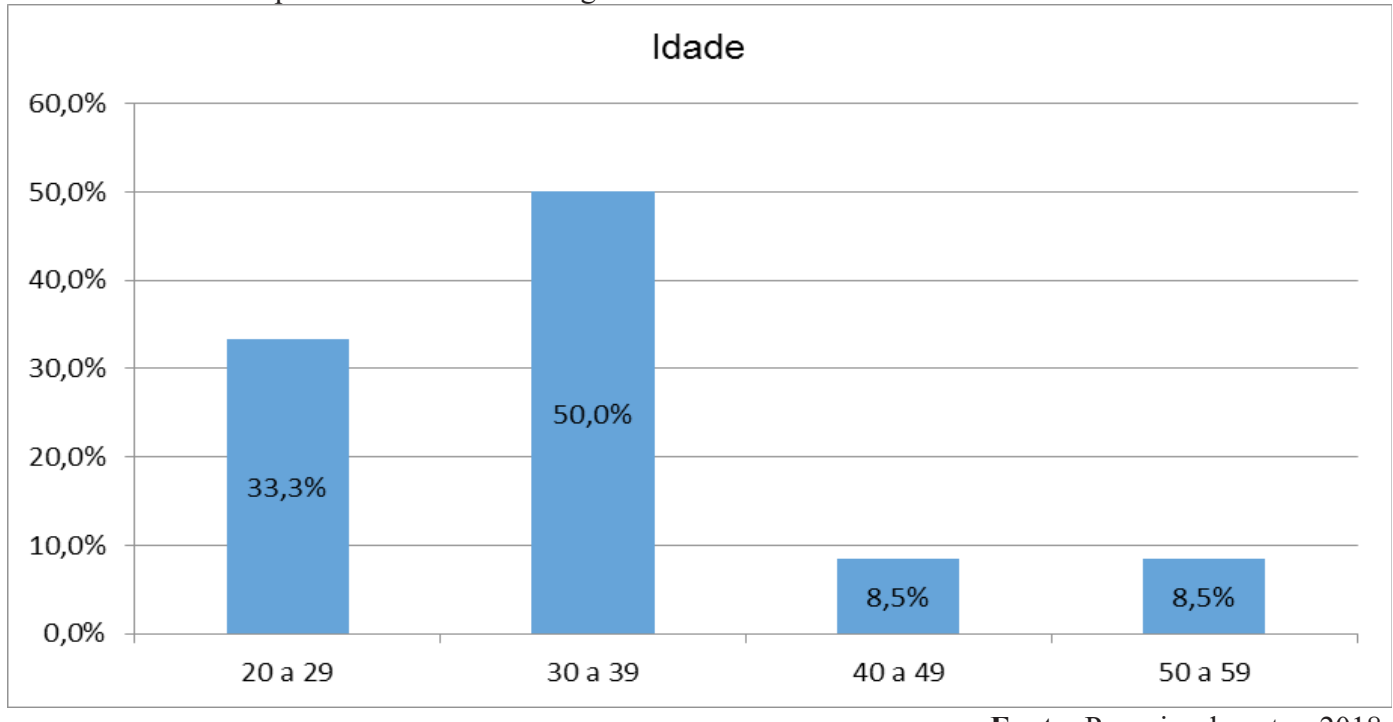

Fonte: Pesquisa do autor, 2018.

Tabela 1. Adesão ao pré-natal no $1^{\circ}$ trimestre nas Unidades de Saúde

\begin{tabular}{cc}
\hline Quantidade de partos & $\%$ \\
\hline Unidade 1 & 38 \\
Unidade 2 & 75 \\
Unidade 3 & 85 \\
Unidade 4 & 73 \\
Unidade 5 & 77 \\
Unidade 6 & 33 \\
Unidade 7 & 100 \\
Unidade 8 & 76 \\
\hline Unidade 9 & 84 \\
Unidade 10 & 88 \\
\hline
\end{tabular}

Fonte: Pesquisa do autor, 2018. 
Muitos são os fatores que podem influenciar a baixa adesão ao pré-natal, principalmente no primeiro trimestre gestacional. Pode citar fatores relacionados à mulher como a dificuldade de reconhecimento e aceitação da gravidez, a falta de apoio na família e também questões relacionadas ao próprio serviço de saúde como a demora no agendamento e falta de profissionais.

\section{Estratégias de captação}

Segundo as participantes, a forma pela qual as mulheres chegam até as unidades, ou seja, a captação, é em sua maioria por meio da procura espontânea. A totalidade das gestantes, $80 \%$, procura o pré-natal no primeiro trimestre gestacional de forma espontânea. Os outros $20 \%$ chegam à unidade por meio da busca ativa. É importante mencionar que essa porcentagem não se refere ao total de gestantes da localidade, mas se refere à parcela que comparece à assistência pré-natal.

Diversas são as estratégias utilizadas na tentativa de atrair as gestantes e sensibilizá-las para a importância da realização da assistência pré-natal no primeiro trimestre gestacional e em todo o período de gravidez. Aqui observa-se a categoria escuta empática, baseado no "intuir empático". A captação pressupõe vínculo, convivência e uma troca constante de saberes e de vivências. $^{2}$

A escuta empática está presente quando a enfermeira tem uma pré-disposição para o cuidado empático, ou seja, quando ela "tem uma pré-disposição para exercer o cuidado que valoriza o sofrimento e a subjetividade do outro, trabalha sobretudo com a sensibilidade, com a emoção e com o afeto". Afirma ainda que esse tipo de postura (ação) acaba por estabelecer "uma relação interpessoal entre quem cuida, com o ser cuidado". ${ }^{3}$

Gráfico 3. Como a gestante chega à unidade

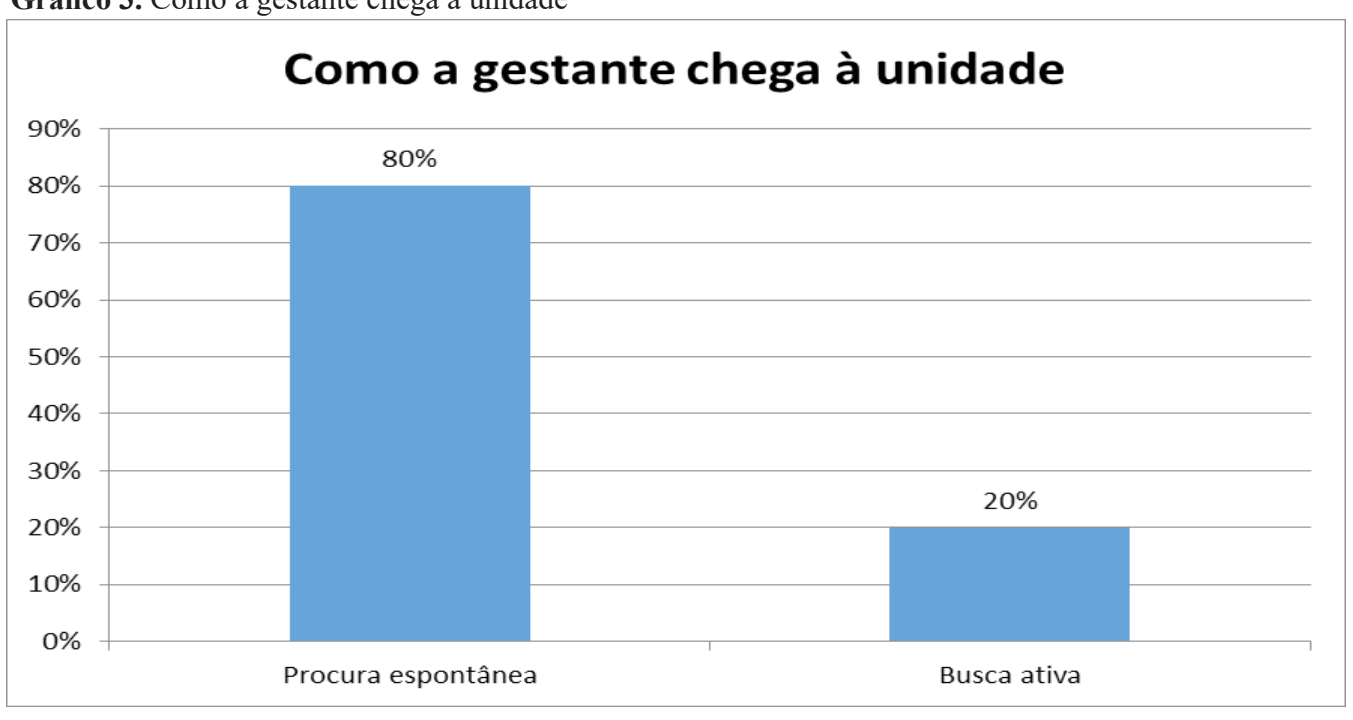

Fonte: Pesquisa do autor, 2018.

O estudo demonstrou que a estratégia mais utilizada pelos enfermeiros e para a captação das gestantes foi a busca ativa, estratégia também que figurou como a mais eficaz. $70 \%$ das enfermeiras participantes utilizaram-se dessa estratégia. Foram utilizadas também como estratégias a comunicação por telefone, mensagens e a realização de reuniões em grupos de educação em saúde.

São raras as unidades onde a procura é total. Mas algumas delas apresentam pouco problemas relevantes.

"Desde a primeira consulta de pré-natal já é feita toda orientação quanto a importância do pré-natal e no momento não tenho a necessidade de captação, elas procuram o atendimento segundo a agenda" (Enfermeira 07)

Pode-se perceber a eficácia da busca ativa como estratégia por meio das seguintes falas:

"A maioria após busca ativa procura a unidade para o início do pré-natal" (Enfermeira 04) "Elas costumam aderir ao pré-natal após busca ativa" (Enfermeira 05) "Na grande maioria das vezes o resultado é positivo, poucas continuam a oferecer resistência" (Enfermeira 06)

Revelam também que após a utilização dessa 
estratégia, se a gestante não comparecer é acionado o Conselho Tutelar, conforme previsto na legislação.

\begin{abstract}
"A maioria dos pré-natais realizados no primeiro trimestre gestacional tem boa adesão e recebem bem a busca ativa (realização dos exames, consultas, imunização). As poucas exceções que não respondem à busca ativa, algumas vezes é necessário acionar o conselho tutelar" (Enfermeira 10)
\end{abstract}

E a busca ativa tem um elemento fundamental. Um elo de ligação entre a unidade e a comunidade que é o Agente Comunitário de Saúde (ACS). Assim como constatou-se nos números que a busca ativa foi a estratégia mais eficaz. Constatou-se também pelas falas das enfermeiras, a relação entre a busca ativa e as ACS que são citadas em $60 \%$ das respostas.

Questionadas sobre as estratégias de captação com as quais trabalham, elas revelam que utilizam:

"Visita dos agentes comunitários. Disponibilidade dos dias
e horários das consultas em local visível" (Enfermeira 06)
"Busca ativa com visita domiciliar dos agentes
comunitários e da enfermeira" (Enfermeira 04)
"Através da busca ativa pelos ACS" (Enfermeira 01)
"Busca ativa através dos ACS, grupo de
educação em saúde" (Enfermeira
"Busca ativa do ACS, visita domiciliar" (Enfermeira 09)
"Busca ativa na comunidade (ACS), orientação em grupo de
saúde da mulher, planejamento familiar" (Enfermeira 10)

A partir desses relatos, fica nítida a importância do papel das ACS na captação das gestantes. São as ACS as portadoras do vínculo com a comunidade e as responsáveis por servirem de ponte entre a comunidade e a unidade/serviço de saúde.

\section{Adaptando-se às necessidades - prontidão para o cuidado}

Um ponto importante de ser mencionado é a flexibilidade das unidades e profissionais de enfermagem em relação a horários e dinâmica de atendimento para que seja possível estar mais próximo da disponibilidade e possibilidade das gestantes. Muda-se o horário, realizase encaixes, abre-se exceções. É muito importante que os profissionais tenham esse "jogo de cintura" visto que em alguns casos não é um capricho ou uma vontade da gestante. É realmente uma impossibilidade de comparecimento. Pode ser uma mudança de datas e horários Conforme as falas a seguir:

"Mudanças na agenda, de acordo com a disponibilidade.
Ou a encaixo no horário do preventivo noturno que
a unidade realiza de 17 às $21 \mathrm{~h}$ " (Enfermeira 06)
"Sempre que necessário abrimos exceções
quanto a dias e horários" (Enfermeira 08)

Algumas demonstram uma vontade imensa de tentar conciliar a agenda de funcionamento da unidade com a disponibilidade da gestante:

"[...] conforme a disponibilidade dela, dentro do horário de funcionamento da Unidade"

"[...]uma adequação de acordo com a disponibilidade da demanda da unidade e a necessidade da gestante".

O estudo apontou que $90 \%$ das enfermeiras demonstrou essa capacidade de adequação. Aqui se identifica uma das categorias mencionadas "quando a enfermeira se disponibiliza para cuidar do indivíduo independentemente da hora e local que ele esteja." É a prontidão para o cuidado, ou quando a enfermeira está apta para cuidar. ${ }^{4}$

A literatura explica que "prontidão para cuidar é estar ao lado do paciente. É estar disponível, fazer um caminho com ele, conhecer e criar com este um espaço de vida". 5

\section{Conclusão}

O estudo realizado possibilitou o entendimento e reconhecimento da necessidade da co-responsabilidade do profissional e da própria gestante nas necessidades da realização das práticas do pré-natal precoce, ampliando o conhecimento do autor sobre o assunto the proporcionando mais segurança e autonomia para trabalhar em rede.

O enfermeiro tem um papel importante no que se refere à assistência pré-natal, principalmente no primeiro trimestre gestacional, pois além da sua função técnica na realização dos procedimentos, tem a missão de estabelecer relação e vínculo com as pacientes gestantes e com as que virão a ser.

Percebeu-se que mais do que dificuldades operacionais ou de contingência, a grande dificuldade que se apresenta aos enfermeiros na atenção básica em relação ao primeiro trimestre gestacional é relacionada aos baixos índices de captação de gestantes para a assistência pré-natal, dificuldades sobretudo na captação precoce.

Em relação às dificuldades dos enfermeiros, foi possível constatar que muitos desses profissionais têm dificuldades inclusive de compreensão dos termos e da política de saúde e das estratégias do país em relação a diminuição dos índices de mortalidade e de aumento da captação no pré-natal. Por fim, o enfermeiro precisa estar capacitado para cuidar, com disponibilidade, mesmo diante das dificuldades.

\section{Referências}

1.Brasil, Ministério da Saúde. Secretaria de Atenção à Saúde. Departamento de Atenção Básica. Cadernos de Atenção Básica, n 32. Atenção ao pré-natal de baixo risco. Brasília; 2012.

2.Cruz RSBLC. Aspectos Históricos, Conceituais e Organizativos do Pré- 
natal, Revista Brasileira de Ciências à Saúde, 2014; 18(1):87-94.

3. Silva GSV. A Incerteza Criativa: O Modelo Teórico do "Intuir Empático" Subsidiando o Cuidado de Enfermagem de Saúde Mental no Programa de Saúde da Família. Dissertação do Mestrado. Universidade Federal do Rio de Janeiro Escola de Enfermagem Anna Nery, 2012.

4. Galavote HS, et al. O trabalho do Enfermeiro na Atenção Primária à Saúde. Escola Ana Nery, [S.L], jan./mai. 2016. Disponível em: http://www.scielo. br/pdf/ean/v20n1/1414-8145-ean-20-01-0090.pdf Acesso em: Outubro de 2018.

5. Nunes ADS, Amador AE, Dantas APQM, Azevedo UM, Barbosa IR. Acesso à assistência pré-natal no Brasil: análise dos dados da pesquisa nacional de saúde. Rev. Bras. Promoção da Saúde. 2017;30(3). 